\title{
EXPERIMENTAL STUDIES OF A CHROMATOGRAPHIC MOVING-BED REACTOR - CATALYTIC OXIDATION OF CARBON MONOXIDE ON ACTIVATED ALUMINA AS A MODEL REACTION -
}

\author{
KAZUO TAKEUCHI AND THE LATE YUZO URAGUCHI \\ Department of Chemical Engineering, \\ University of Tokyo, Tokyo 113
}

\begin{abstract}
The chromatographic moving-bed reactor is a new device which possesses functions of both reactor and adsorptive separator. This paper demonstrates experimentally that reaction and separation occur simultaneously in the device for the oxidation of carbon monoxide over activated alumina with first-order irreversible kinetics.

The observed concentration profiles of the reactant and the product along the longitudinal direction of the bed are compared with the theoretical curves to show that the proposed model is valid. The effect of non-linearity of adsorption isotherms for the product at high surface concentration is also discussed.
\end{abstract}

\section{Introduction}

Industrial reaction processes are almost always followed by separation processes. Most process engineers have been, therefore, oriented toward optimizing possible combinations of known reaction and separation processes. On the other hand, only a few attempts have been made to propose new processes which possess these two functions in one apparatus. Distillation accompanied by homogeneous chemical reaction and parametric pumping with heterogeneous catalytic reaction are examples. In previous papers ${ }^{3,4)}$, the authors proposed a new combined process of adsorptive separation and catalytic reaction, called a chromatographic moving-bed reactor. In the new reactor, the product formed on catalyst surface is continuously separated from the unreacted component utilizing differences between adsorptivities of the reactant and the product. Though simplification and size reduction of the reactorseparator system is the primary significance of this process, its potential applications lie in improving the yield of the desired product in reversible or consecutive reactions such as of reactive distillation.

This process can also be considered for continuous large-scale operation of the chromatographic (pulse) reactor in chemical laboratories where reaction and adsorptive separation take place in unsteady-state fashion. This fixed-bed chromatographic technique has been applied to various reaction schemes such as

Received on September 3, 1976. Correspondence concerning this article should be addressed to $\mathrm{K}$. Takeuchi.
$A \longrightarrow B, A \rightleftharpoons B$, and $A+B \rightleftharpoons C$ as Langer et al. ${ }^{11}$ pointed out in their comprehensive review.

Similarly, the chromatographic moving-bed reactor can be applied to any reaction scheme mentioned above. It is, however, necessary first to investigate a fundamental case such as first-order irreversible reaction in order to show the validity of such a new process experimentally.

As in the case of reactive distillation, an exhausting section is required to recover the unreacted component adsorbed on catalyst surface as is discussed elsewhere ${ }^{5)}$.

Only the rectifying section of the chromatographic moving-bed reactor is discussed in this paper, however, because analysis of the exhausting section differs from that of the rectifying section only in the boundary conditions.

\section{Experimental}

\section{1 Catalyst/adsorbent}

Activated alumina KHA-46 from Sumitomo Chemical Co. for catalytic oxidation of carbon monoxide was chosen for the following reasons: (1) it exhibits first-order kinetics with no noticeable decay of catalyst activity during the experiments, (2) the reaction rate is moderate enough to make the assumption of isothermal conditions valid; (3) the difference between adsorptivity of the reactant and that of the product is quite large; and (4) it has good mechanical strength for the use in the moving bed.

Adsorption equilibrium constants, adsorption rate constants and intraparticle diffusivities for carbon 


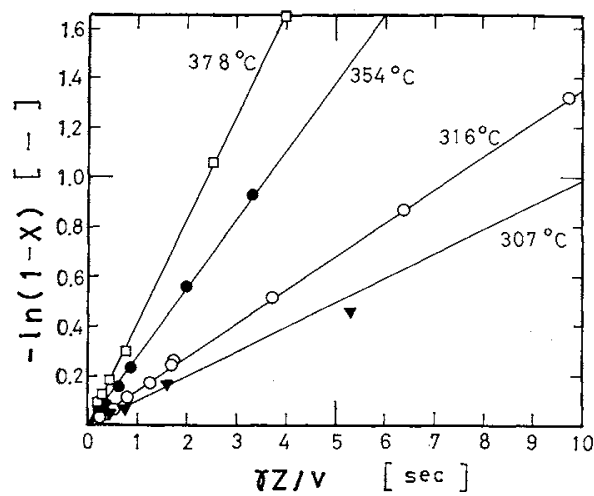

Fig. 1 Experimental results for fixed bed reactor (conversion vs. contact time)

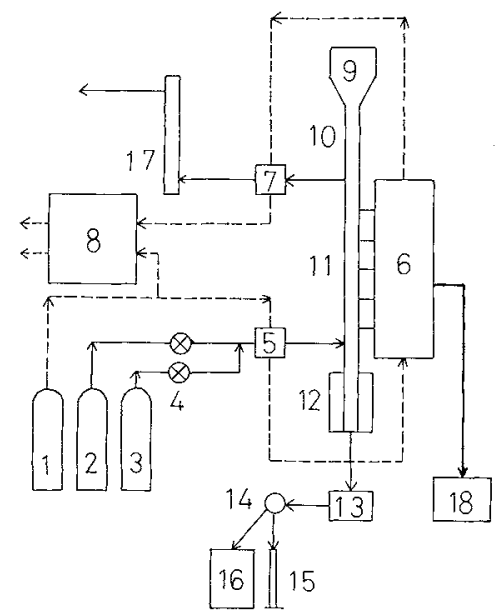

1. Helium cylinder $(99.995 \%)$

2. Cylinder of mixed gas of oxygen $(20.1 \%)$ and helium

3. Carbon monoxide cylinder $(99.5 \%)$

4. Mass-flow valve

5. Sampling valve for inlet gas stream

6. Simultaneous gas sampling device

7. Sampling valve for outlet gas stream

8. Gas chromatograph

9. Catalyst hopper $(2000 \mathrm{ml})$

10. Preheating section $(30 \mathrm{~cm})$

11. Test section $(55 \mathrm{~cm})$

12. Water-jacketed cooling section $(30 \mathrm{~cm})$

13. Vibratory feeder

14. Solid sampling valve

15. Glass vessel for solid flow rate measurement

16. Catalyst reservoir

17. Soap film flow meter

18. Vacuum pump

Fig. 2 Schematic diagram of experimental apparatus

monoxide and carbon dioxide were reported in the previous paper $^{6)}$ along with physical properties of the catalyst.

\section{2 Surface reaction rate constant}

Gas mixture of $\mathrm{CO}(0.53 \%), \mathrm{O}_{2}(20.1 \%)$ and helium (balance) was introduced to a steady-state laboratory fixed-bed reactor (i.d. $4 \mathrm{~mm} \times 10.0 \mathrm{~cm}$ ) packed with catalyst of $48-70$ mesh at $307^{\circ} \mathrm{C}-378^{\circ} \mathrm{C}$. Measurements of inlet and outlet concentration of carbon monoxide were made with a gas chromatograph equipped with a silica gel column (i.d. $3 \mathrm{~mm} \times 100 \mathrm{~cm}$ ) at $40^{\circ} \mathrm{C}$.

Logarithmic plots of fractional non-conversion versus contacting time show straight lines as shown in Fig. 1, indicating first-order kinetics. Surface reaction rate constant $k_{r}$ was obtained from the slopes of the lines in Fig. 1 and the following relations obtained by Suzuki and $\mathrm{Smith}^{2)}$ using a one-dimensional Fibonacci search.

$$
\begin{aligned}
& 1-X=\exp \left\{-v\left(q_{0}-1\right) Z /\left(2 E_{A}\right)\right\} \\
& q_{0}=\left\{1+4 E_{\alpha} G(0) / v^{2}\right\}^{1 / 2} \\
& G(0)=-\frac{3(1-\alpha) B i}{\alpha R^{2} / D_{i}}\left[1-\frac{B i}{A_{0}(0)+B i}\right] \\
& A_{0}(0)=\phi(0) \operatorname{coth} \phi(0)-1 \\
& \phi(0)=R\left[\frac{\beta}{D_{i}\left\{\left(1 / k_{a F}\right)+\left(1 / k_{r} K_{F}\right)\right\}}\right]^{1 / 2}
\end{aligned}
$$

Arrhenius-type description of surface reaction rate constant is given by

$$
k_{r}=5.33 \times 10^{4} \exp \left(-14700 / R_{g} T\right)
$$

The values of adsorption equilibrium constant and adsorption rate constant for carbon monoxide over activated alumina were adopted from the previous work $^{6}$. Average tortuosity factor for intraparticle diffusion was found to be 2.83. Sherwood number was assumed to be 2. Axial dispersion coefficient was calculated by the correlation of Wen and $\operatorname{Fan}^{7)}$. The effectiveness factor is greater than 0.99 ; it is defined by

$$
E . F .=3[\phi(0) \operatorname{coth} \phi(0)-1] /[\phi(0)]^{2}
$$

1. 3 Description of the moving-bed reactor and experimental procedure

A schematic diagram of the moving-bed reactor is shown in Fig. 2. The moving bed is made of stainless steel with an inner diameter of $14.0 \mathrm{~mm}$. The rectifying section is $55 \mathrm{~cm}$ long, and the locations of gas sampling are $5 \mathrm{~cm}, 15 \mathrm{~cm}, 25 \mathrm{~cm}, 35 \mathrm{~cm}$, and $45 \mathrm{~cm}$ above the feed location.

Simultaneous gas sampling at these locations is necessary because sequential sampling procedure inevitably disorders the concentration profiles in the axial direction of a moving-bed reactor of this size. The apparatus was initially filled with a gas mixture of oxygen $(20.1 \%$ ) and helium (balance) and the catalyst surface was saturated with oxygen. During the operation, the apparatus was made airtight except at the gas inlet and outlet. Gas chromatograph with TCD detectors equipped with double columns of silica gel (i.d. $3 \mathrm{~mm} \times 100 \mathrm{~cm}$ ) and molecular sieves $5 \mathrm{~A}$ (i.d. $3 \mathrm{~mm} \times 200 \mathrm{~cm}$ ) was used to analyse $\mathrm{CO}, \mathrm{CO}_{2}$ and $\mathrm{O}_{2}$ at $40^{\circ} \mathrm{C}$. The feed was a mixture of carbon monoxide $(5.5 \%)$, oxygen $(19.0 \%)$ and helium (balance). It took 2 to 5 hours for the reactor to reach steady state. The catalyst particles of 40-60 mesh used were regenerated in a fixed bed at $430^{\circ} \mathrm{C}$ for 48 hours to 


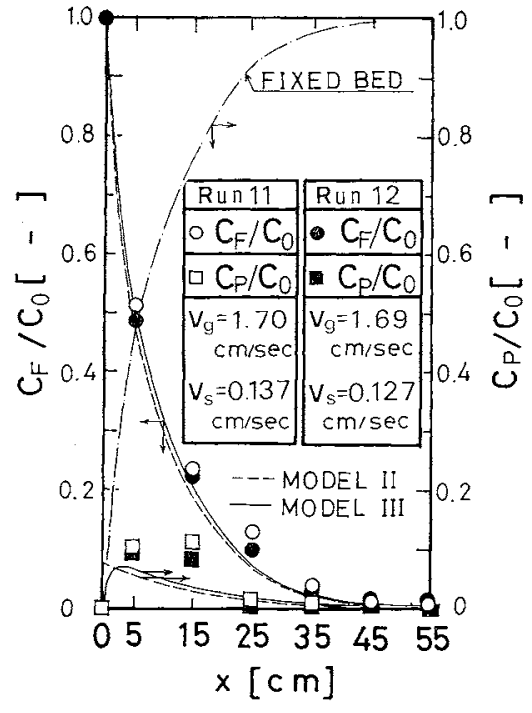

Fig. 3 Comparison of experimental concentration profiles at $341^{\circ} \mathrm{C}$ with theoretical prediction

desorb carbon dioxide from the surface. All experimental runs were carried out at $341^{\circ} \mathrm{C}$ and under atmospheric pressure. Maximum pressure drop across the test section did not exceed $3 \mathrm{~cm} \mathrm{Hg}$. The values for $v_{g}$ and $v_{s}$ were so chosen that $v_{g} / v_{s}$ was within the separation conditions shown in the previous work $\mathrm{k}^{3}$.

\section{4 Experimental results}

Typical concentration profiles of the reactant and the product in the chromatographic moving-bed reactor are shown in Figs. 3 to 6 . Also shown in Fig. 3 is the theoretical concentration profile of the product in a fixed bed reactor operated under the same conditions as the moving-bed reactor except for $v_{s}$, which is set at zero. The concentration profile of the product in a fixed bed reactor can be safely predicted from the measured reaction rate constant in Eqs. (1) to (6) and the simple mass balance relation $C_{F}+C_{P}=C_{0}$ for $0 \leqq x \leqq Z$. The difference between $C_{P 1}\left(C_{P}\right.$ at $\left.x=Z\right)$ in the fixed bed and $C_{P 1}$ in the moving bed shows that reaction and separation are actually occurring simultaneously in this apparatus. Runs 11 and 12 in Fig. 3 are performed for reproducibility checks. The void fraction of the moving bed, $\alpha$, is assumed to be the same as that of a randomly packed bed of the same geometry, namely, $\alpha=0.40$.

\section{Interpretation of the Results Based on Linear Ad- sorption Isotherms}

To formulate a model, the following assumptions are made: (1) isothermal operation, (2) constant linear gas and solid velocities in the reactor, (3) firstorder irreversible reaction with respect to surface concentration of the reactant, (4) linear adsorption isotherms, (5) negligible axial dispersion in both fluid and solid phases and (6) continuous steady-state operation of the whole system.

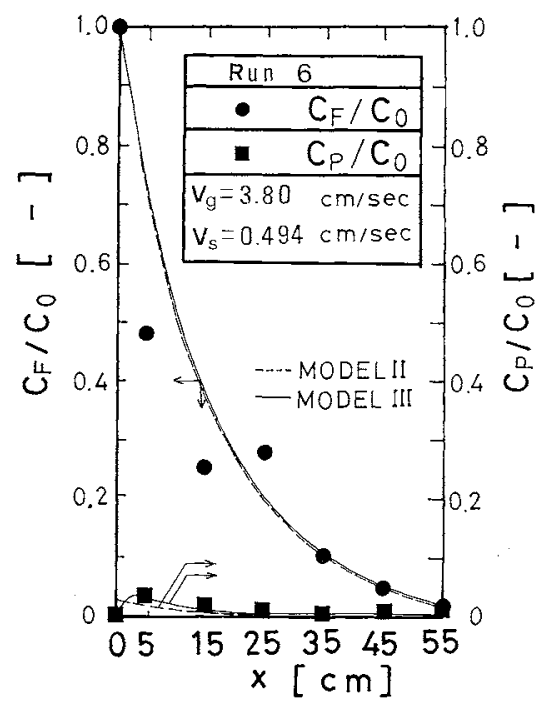

Fig. 4 Comparison of experimental concentration profiles at $341^{\circ} \mathrm{C}$ with theoretical prediction

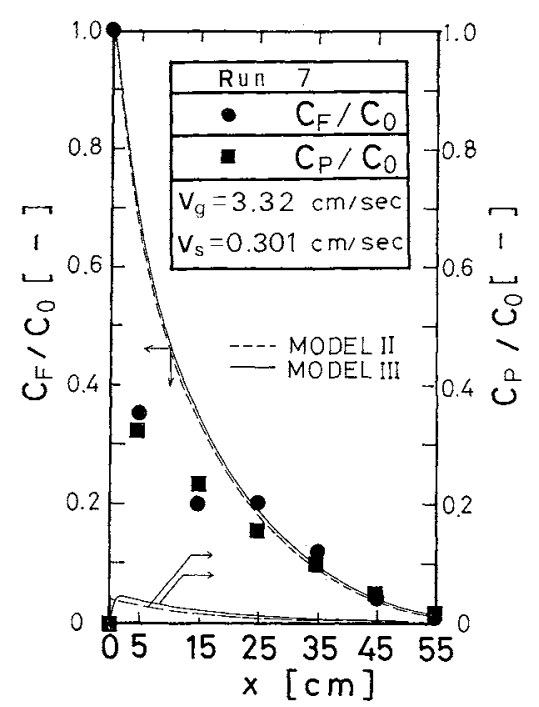

Fig. 5 Comparison of experimental concentration profiles at $341^{\circ} \mathrm{C}$ with theoretical prediction

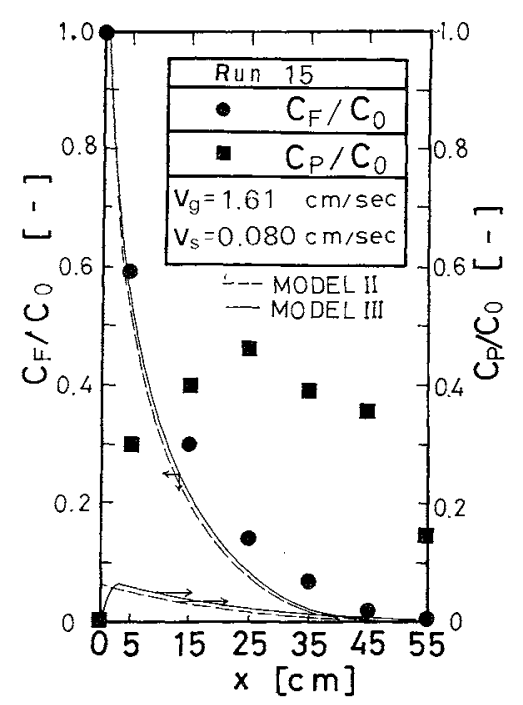

Fig. 6 Comparison of experimental concentration profiles at $341^{\circ} \mathrm{C}$ with theoretical prediction 


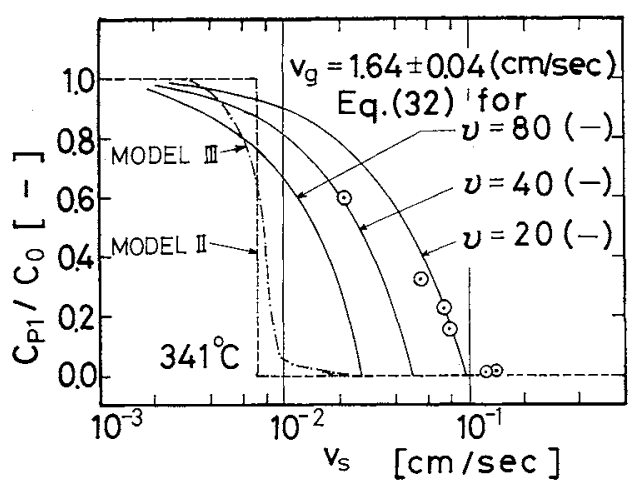

Fig. 7 Outlet concentration of the product $C_{P 1}$ vs. $v_{s}$ when $v_{g}$ is fixed

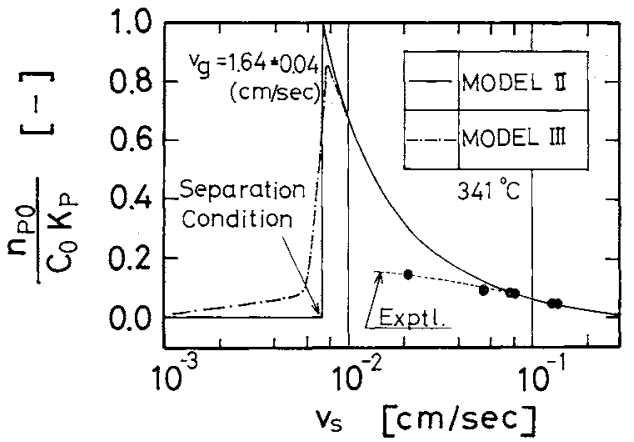

Fig. 8 Surface concentration of the product at $x=0$ vs. $v_{s}$ when $v_{g}$ is fixed

From these assumptions, the following mathematical model is proposed along with three kinds of parameters, i.e., adsorption equilibrium constants $\left(K_{F}\right.$ and $\left.K_{P}\right)$, adsorption rate constants $\left(k_{a F}\right.$ and $\left.k_{a P}\right)$, and surface reaction rate constant $\left(k_{r}\right)$. This model is named Model III after the number of kinds of parameters. In Model III, there are no concentration gradients in fluid film and pore spacing with respect to the radial direction of the particles. Pointwise adsorption equilibrium between the surface and pore spacing is assumed not to be attained.

Under these condtions, basic equation of the rectifying section for the reactant and the product are given by

$$
\begin{aligned}
& \left(v_{g}-\gamma v_{s}\right)\left(d C_{F} / d x\right)+\gamma\left(k_{a F} C_{F}-k_{a F} n_{F} / K_{F}\right)=0 \\
& v_{s}\left(d n_{F} / d x\right)+k_{a F} C_{F}-\left(k_{r}+k_{a F} / K_{F}\right) n_{F}=0 \\
& \left(v_{g}-\gamma v_{s}\right)\left(d C_{P} / d x\right)+\gamma\left(k_{a P} C_{P}-k_{a P} n_{P} / K_{P}\right)=0 \\
& v_{s}\left(d n_{P} / d x\right)+k_{a P}\left(C_{P}-n_{P} / K_{P}\right)+k_{r} n_{F}=0
\end{aligned}
$$

The velocity terms in Eqs. (8) and (10) are $\left(v_{g}-\gamma v_{s}\right)$ because of the effect of the pore volume and the relation $C_{J}=C_{i J}$.

The boundary conditions are written as follows:

$$
\begin{array}{ll}
C_{F}=C_{0} & \text { at } x=0 \\
n_{F}=0 & \text { at } x=Z \\
C_{P}=0 & \text { at } x=0 \\
n_{P}=0 & \text { at } x=Z
\end{array}
$$

Table 1 Discontinuities of concentration profiles of reactant and product in Model III $(J=F$ or $P)$

\begin{tabular}{cccc} 
& $C_{J}$ & $C_{i j}$ & $n_{J}$ \\
\hline$x=Z^{*}$ & $C_{J 1}^{*}=\left(\frac{v_{g}-r v_{s}}{v_{g}}\right) C_{J 1}$ & 0 & 0 \\
$x=Z$ & $\begin{array}{c}C_{J 1} \\
\text { setting } x=Z \text { in } \\
\text { Eqs. (16) and (22) }\end{array}$ & $C_{i J 1}=C_{J 1}$ & $n_{J 1} \neq K_{J} C_{i J}$ \\
& & Eqs. (17) and (23)
\end{tabular}

Table 2 Special cases of Model III for reactant

\begin{tabular}{cccc}
$\begin{array}{c}\text { Limiting } \\
\text { cases }\end{array}$ & $\begin{array}{c}\text { Effectiveness } \\
\text { factor }\end{array}$ & \multicolumn{1}{c}{$\frac{C_{F 1} \text { (Model II) }}{C_{F 1} \text { (Model III) }} \frac{n_{F 0} \text { (Model III) }}{n_{F 0} \text { (Model II) }}$} \\
\hline$\Psi \rightarrow 0$ & 1.0 & 1.0 & 1.0 \\
$K_{F} \rightarrow 0$ & $\frac{1}{1+\Psi}$ & $\exp \left(\frac{\Psi}{1+\Psi}\right)$ & $\frac{1}{1+\Psi}$ \\
\hline
\end{tabular}

The $x$-coordinate is taken as positive in the direction of fluid flow. The solutions of the basic equations (8) and (9) for the reactant are given by

$$
\begin{aligned}
& C_{F} / C_{0}=A_{1} \exp \left(\lambda_{1} \xi\right)+A_{2} \exp \left(\lambda_{2} \xi\right) \\
& n_{F} /\left(C_{0} K_{F}\right)=B_{1} \exp \left(\lambda_{1} \xi\right)+B_{2} \exp \left(\lambda_{2} \xi\right)
\end{aligned}
$$

The constants of integration are given in Appendix 1. Discontinuities of the concentration profiles exist at $x=Z$, because $C_{J}=C_{i J}$ for $0<x<Z$ and $C_{i J}^{*}=0$ at $x=Z^{*}$, while $C_{i J} \neq 0$ at $x=Z$. They are summarized in Table 1.

The characteristic equation with two real roots $\lambda_{1}$ and $\lambda_{2}$ is given by

$$
K_{F} \Psi \lambda^{2}+\Omega\left\{\frac{K_{F} b}{1-b}-(1-\Psi)\right\} \lambda-\Omega^{2}\left(\frac{b}{1-b}\right)=0
$$

where dimensionless constants are given by

$$
\begin{aligned}
& \Psi=k_{r} K_{F} / k_{a F} \\
& \Omega=k_{r} K_{F} Z / v_{s} \\
& b=\gamma v_{s} / v_{g}
\end{aligned}
$$

The characteristic equation (18) is reduced to a firstorder equation for the following special cases. When $\Psi$ approaches zero, $\lambda$ approaches $\lambda_{s}=\gamma k_{r} K_{F} Z /\left\{\gamma v_{s}(1+\right.$ $\left.\left.K_{F}\right)-v_{g}\right\}$, which is the single pole of the simplest model described in the previous paper ${ }^{4}$. This model is a limiting case of the present Model III with $k_{a F}$, $k_{a P} \rightarrow \infty$; it is proper to call this simple case Model II.

When $K_{F}$ approaches zero, $\lambda$ approaches $\lambda_{s} /(1+\Psi)$. The term $1 /(1+\Psi)$ is an effectiveness factor for finite adsorption rate constant as shown by Suzuki and Smith $^{2}$. These relations are summarized in Table 2. On the other hand, the solutions for the product are given by

$$
\begin{aligned}
& C_{P} / C_{0}=C_{1} e^{\kappa \xi}+C_{2}+C_{3} e^{\lambda_{1} \xi}+C_{4} e^{\lambda_{2} \xi} \\
& n_{P} /\left(C_{0} K_{P}\right)=D_{1} e^{\alpha \hat{\xi}}+D_{2}+D_{3} e^{\lambda_{1} \xi}+D_{4} e^{\lambda_{2} \xi}
\end{aligned}
$$

Constants of integration are shown in Appendix 2. The third pole for the product concentration profiles is given by

$$
\kappa=\Phi\left\{\begin{array}{c}
b\left(1+K_{P}\right)-1 \\
b-1
\end{array}\right\}
$$


where

$$
\Phi=k_{a P} Z / v_{s}
$$

\section{Discussion}

As shown in Figs. 3 to 6, the difference between the theoretical (from Model II and III) and the experimental results for the reactant concentration lies within the range of the reactivity deviation of repeatedly-regenerated catalyst from run to run.

Consideration of the accuracy of the results is required with regard to the use of extrapolated values for $k_{a F}$ and $K_{F}$ obtained below $165^{\circ} \mathrm{C}$ in the previous investigation ${ }^{6}$. Since $C_{F^{\prime 1}}$ (Model III) is close to $C_{F \perp}$ (Model II) as long as $1 /\left(1+\Psi^{\prime}\right)$ is sufficiently close to 1 , as shown in Table 2 and $\Psi \ll 1$, inaccuracy of $k_{a F}$ associated with extrapolation can be considered insignificant. Furthermore, because the operating conditions are so chosen that $v_{g}$ is considerably greater than $v_{s}$, the error associated with extrapolated $K_{F}$ in the term of $\left\{v_{g}-\gamma v_{s}\left(1+K_{F}\right)\right\}$ is also insignificant.

For the product, the slowest mass transfer stage is found to be the adsorption rate because $\delta_{a} \gg \delta_{D} \gg \delta_{f}$ under the experimental conditions, where the contributions of adsorption rate constant, intraparticle diffusivity and film mass transfer coefficient in second central moment for impulse response in adsorption chromatography ( $\delta_{a}, \delta_{D}$, and $\delta_{f}$, respectively) are written $\mathrm{as}^{2 \text { ? }}$

$$
\begin{aligned}
& \delta_{a}=2 \gamma K_{P}^{2} / k_{a P} \\
& \delta_{D}=2 \gamma\left(1+K_{P}\right)^{2} R^{2} \beta /\left(15 D_{i P}\right) \\
& \delta_{f}=2 \gamma\left(1+K_{P}\right)^{2} R \beta /\left(3 k_{f P}\right)
\end{aligned}
$$

The agreement between the theoretical and experimental profiles for the product in Figs. 3 and 4 are good when $v_{g} / v_{s}$ is relatively small.

Model III with finite mass transfer rate for the product can predict the non-zero values for $C_{P 1}$ at $x=Z$, while Model II with $k_{a P} \rightarrow \infty$ cannot. Model III is, however, not sufficient for the cases shown in Figs. 5 and 6 . This discrepancy will be discussed in the next section.

\section{Interpretation of the Results with Nonlinear Ad- sorption Isotherm}

Figure 7 shows the results of outlet concentration of the product versus $v_{s}$, when $v_{g}$ is fixed. Figure 8 shows the experimental values for $n_{P 0} / C_{0} K_{P}$ calculated from a simple mass balance equation given by

$$
\gamma v_{s} n_{P 0}=C_{0}\left(v_{g}-\gamma v_{s}\right)\left\{1-\exp \left(\lambda_{s}\right)\right\}-\left(v_{g}-\gamma v_{s}\right) C_{P 1}
$$

They tend to deviate from the theory for the case of linear adsorption isotherm and to level off as $v_{s}$ decreases. In other words, the experimental $n_{P 0} / C_{0} K_{P}$ asymptotically approaches saturation at high surface coverage, which implies nonlinear isotherm such as the Langmuir type.

To describe the whole concentration profile for the product with nonlinear isotherm, a two-point bounary value problem for a pair of nonlinear differential equations must be solved. Though such a numerical calculation is beyond the scope of this report, the behaviour for $C_{P 1}$ can be discussed as follows.

Let adsorption isotherm for the product be assumed as Langmuir type, written as

$$
n_{P}=\theta N=K_{P} C_{P} /\left\{1+\left(C_{P} K_{P} / N\right)\right\}
$$

where $N$ is the concentration of adsorption sites and $\theta$ is the surface coverage of the product. Since $K_{F}$ is much smaller than $K_{P}$, the surface coverage of the reactant can be neglected.

Maximum surface coverage of the product $\theta_{\max }$ is given by

when

$$
\theta_{\max }=K_{P} /\left(K_{P}+\nu\right)
$$

where

$$
C_{P}=C_{0}
$$

$$
\nu=N / C_{0}
$$

When $\theta$ is close to $\theta_{\max }$, the mass balance equation described below holds.

$$
\begin{aligned}
& C_{0}\left(v_{g}-\gamma v_{s}\right)\left\{1-\exp \left(\lambda_{s}\right)\right\}=C_{P 1}\left(v_{g}-\gamma v_{s}\right)+\gamma n_{P 0} v_{s} \\
& \quad=C_{P 1}\left(v_{g}-\gamma v_{s}\right)+\gamma v_{s} N K_{P} /\left(\nu+K_{P}\right)
\end{aligned}
$$

The theoretical curves for $C_{P 1} / C_{0}$ calculated from Eq. (32) for given $\nu$ are also shown in Fig. 7. Since the effect of finite mass transfer rate is neglected, these curves (solid lines) intersect the abscissa instead of approaching it asymptotically as $v_{s}$ increases, as in the case of Model III. This may be the reason why the experimental data in Fig. 7 do not exactly lie on a certain theoretical curve.

Nevertheless, such a simplified correction of isotherms is helpful in the qualitative explanation of the change of experimental $C_{P 1} / C_{0}$ with the change of $v_{s}$. Namely observed $C_{P 1} / C_{0}$ decreases at larger $v_{s}$ than predicted from Model III.

\section{Conclusions}

1) It was confirmed that reaction and separation were actually taking place in this reactor/separator.

2) The concentration profiles for the reactant can be interpreted theoretically based on a linear adsorption isotherm.

3) Deviation of the product from the theory with linear isotherm is observed when $v_{g} / v_{s}$ is relatively large. This is attributed to nonlinearity of adsorption isotherms.

Appendix 1: Constants of integration for the reactant in Eqs. (16) and (17)

$$
A_{1}=\left[1-\left\{\frac{(1-b) \lambda_{1}+\Omega b / \Psi}{(1-b) \lambda_{2}+\Omega b / \Psi}\right\} e^{\left(\lambda_{1}-\lambda_{2}\right) \xi}\right]^{-1}
$$




$$
\begin{aligned}
& A_{\mathbf{2}}=1-A_{1} \\
& B_{1}=\left\{1+(1-b) \lambda_{1} \Psi /(\Omega b)\right\} A_{1} \\
& B_{2}=\left\{1+(1-b) \lambda_{2} \Psi /(\Omega b)\right\} A_{2}
\end{aligned}
$$

Appendix 2: Constants of integration for the product in Eqs. (22) and (23)

$$
\begin{aligned}
& C_{3}=-\frac{B_{1}}{\kappa}\left(\frac{1}{\lambda_{1}-\kappa}-\frac{1}{\lambda_{1}}\right)\left(\frac{\Omega \Phi b}{1-b}\right) \frac{1}{K_{P}} \\
& C_{4}=-\frac{B_{2}}{\kappa}\left(\frac{1}{\lambda_{2}-\kappa}-\frac{1}{\lambda_{2}}\right)\left(\frac{\Omega \Phi b}{1-b}\right) \frac{1}{K_{P}} \\
& C_{1}= {\left[C_{3}\left\{\frac{(1-b) \lambda_{1}}{b \Phi}\right\} e^{\lambda_{1}}+C_{4}\left\{\frac{(1-b) \lambda_{2}}{b \Phi}\right\} e^{\lambda_{2}}\right] } \\
& \times\left[1-\left\{1+\frac{(1-b) \kappa}{b \Phi}\right\} e^{\kappa}\right] \\
& C_{2}=-C_{1}-C_{3}-C_{4} \\
& D_{1}= C_{1}\left\{\frac{(1-b) \kappa}{b \Phi}+1\right\} \\
& D_{2}= C_{2} \\
& D_{3}= C_{3}\left\{\frac{(1-b) \lambda_{1}}{b \Phi}+1\right\} \\
& D_{4}= C_{4}\left\{(1-b) \lambda_{2}\right. \\
& b \Phi \\
&
\end{aligned}
$$

\section{Nomenclature}

$A_{1}, A_{2}=$ defined by Eqs. (A-1) and (A-2) in Appendix 1

$A_{0}(0) \quad=$ defined by Eq. (4)

$B_{1}, B_{2} \quad=$ defined by Eqs. (A-3) and (A-4) in Appendix 1

$\mathrm{Bi} \quad=k_{f} R / D_{i}$, Biot number

$b \quad=$ defined by Eq. (21)

C $=$ concentration in bulk phase

$C_{1}, C_{2}, C_{3}, C_{4}=$ defined by Eqs. (A-5) to (A-8) in Appendix 2

$C_{i} \quad=$ concentration in pore spacing

$C_{0} \quad=$ inlet concentration of reactant

$D_{1}, D_{2}, D_{3}, D_{4}=$ defined by Eqs. (A-9) to (A-12) in Appendix 2

$D_{A B} \quad=$ molecular diffusivity

$D_{i} \quad=$ effective intraparticle diffusivity

$E_{A} \quad=$ axial dispersion coefficient

E.F. $\quad=$ effectiveness factor defined by Eq. (7)

$G_{0} \quad=$ defined by Eq. (3)

$K=$ adsorption equilibrium constant based on unit pore volume

$k_{a} \quad=$ adsorption rate constant based on unit pore volume

$k_{f}=$ film mass transfer coefficient

$[1 / \mathrm{sec}]$

$[\mathrm{cm} / \mathrm{sec}]$

$=$ first-order surface reaction rate constant $[1 / \mathrm{sec}]$

$=$ concentration of adsorption sites based on unit pore volume

$\left[\mathrm{mol} / \mathrm{cm}^{3}\right]$

$=$ surface concentration of adsorbed

$\left[\mathrm{mol} / \mathrm{cm}^{3}\right]$ $\left[\mathrm{mol} / \mathrm{cm}^{3}\right]$

$$
[-]
$$

$\left[\mathrm{cm}^{2} / \mathrm{sec}\right]$

$\left[\mathrm{cm}^{2} / \mathrm{sec}\right]$

$\left[\mathrm{cm}^{2} / \mathrm{sec}\right]$ species based on unit pore volume $\quad\left[\mathrm{mol} / \mathrm{cm}^{3}\right]$

$=$ defined by Eq. (2) $[-]$

$=$ average particle radius $\quad[\mathrm{cm}]$

$=$ gas constant $\quad\left[\mathrm{cal} / \mathrm{mol}-{ }^{\circ} \mathrm{K}\right]$

$=$ Sherwood number, $2 k_{f} R / D_{A B}$

$=$ interparticle linear gas velocity in fixed bed

$=$ interparticle linear gas velocity in moving bed

$=$ linear solid velocity in moving bed $\quad[\mathrm{cm} / \mathrm{sec}]$

= fractional conversion

$=$ axial distance from gas inlet, positive in direction of gas flow

$[-]$

$=$ length of test section

[cm]

[cm]

$=$ void fraction of bed

$=$ porosity of catalyst

$=(1-\alpha) \beta / \alpha$

$=$ surface coverage of the product [-]

$=$ defined by Eq. (24) [-]

$=$ roots of Eq. (18)

$=\gamma k_{r} K_{F} Z /\left\{\gamma v_{s}\left(1+K_{F}\right)-v_{g}\right\}$, pole of Model II [-]

- second central moment for impulse response in adsorption chromatography $\left[\mathrm{sec}^{2}\right]$

$=N / C_{0}$

$=$ dimensionless length along axis of moving bed, $x / Z$

$\Phi \quad=$ defined by Eq. (25) $\quad[-]$$$
\xi
$$

$\phi(0) \quad=$ Thiele modulus defined by Eq. (5) $\quad[-]$

$[-]$

[-] $\Psi \quad=$ defined by Eq. (19) [-]

[-] $Q \quad=$ defined by Eq. (20) [-]

[-] 〈Subscripts〉

$F$
$J$
$P$
0
1

$$
\begin{aligned}
& =\text { reactant } \\
& =\text { arbitrary component } \\
& =\text { product } \\
& =\text { at } x=0 \\
& =\text { at } x=Z
\end{aligned}
$$

$\langle$ Superscript〉

$$
\text { * } \quad=\text { refers to the position just outside }
$$
the test section

\section{Literature Cited}

1) Langer, S. H. et al.: Ind. Eng. Chem., 61, 11 (1969).

2) Suzuki, M. and J. M. Smith: Chem. Eng. Sci., 26, 221 (1971).

3) Takeuchi, K. and Y. Uraguchi: I. Chem. Eng. Japan, 9, 164 (1976).

4) idem.: ibid., 9, 246 (1976).

5) idem.: ibid., 10, 72 (1977).

6) idem.: ibid., 10, 297 (1977).

7) Wen, C. Y. and L. T. Fan: "Models for Flow Systems and Chemical Reactors", pp. 167-174, Marcel Dekker Inc., New York (1975). 